\title{
Copy number analysis identifies tumor suppressive IncRNAs in human osteosarcoma
}

\author{
JIANJUN XIE ${ }^{1,2}$, DECHEN LIN ${ }^{1}$, DHONG HYUN TONY LEE ${ }^{1}$, JENNIFER AKUNOWICZ ${ }^{3}$, MARC HANSEN $^{3}$, \\ CARL MILLER $^{1}$, MASASHI SANADA ${ }^{4}$, MOTOHIRO KATO $^{4}$, TADAYUKI AKAGI ${ }^{1}$, \\ NORIHIKO KAWAMATA ${ }^{1}$, SEISHI OGAWA ${ }^{4}$ and H. PHILLIP KOEFFLER ${ }^{1,5,6}$ \\ ${ }^{1}$ Division of Hematology and Oncology, Cedars-Sinai Medical Center, UCLA School of Medicine, Los Angeles, \\ CA 90048, USA; ${ }^{2}$ Department of Biochemistry and Molecular Biology, Medical College of Shantou University, \\ Shantou, Guangdong 515041, P.R. China; ${ }^{3}$ Center for Molecular Medicine, University of Connecticut Health Center, \\ Farmington, CT 06030-3101, USA; ${ }^{4}$ Pathology and Tumor Biology, Kyoto University, Kyoto 606-8315, Japan; \\ ${ }^{5}$ Cancer Science Institute of Singapore, National University of Singapore, Singapore 117599; \\ ${ }^{6}$ National University Cancer Institute, National University Health System and \\ National University of Singapore, Singapore 119074, Republic of Singapore
}

Received September 12, 2016; Accepted January 23, 2017

DOI: $10.3892 /$ ijo.2017.3864

\begin{abstract}
Osteosarcoma(OS) has a high degree of chromosomal instability and total copy number $(\mathrm{CN})$ changes. We examined 58 human OS samples including 40 primary tumors, 11 explants, and 7 cell lines using single nucleotide polymorphism (SNP) arrays, and revealed that $70 \%$ of the samples had one or more recurrent $\mathrm{CN}$-neutral loss of heterozygosity (CNN-LOH) also known as uniparental disomy (UPD). Importantly, 17\% of the samples showed prominent homozygous deletion of $3 q 13.31$, suggesting its role in tumorigenesis. We identified and characterized two novel lncRNAs, LOC285194 and BC040587, within this genomic locus, strongly suggesting their tumor suppressor activity. Frequent deletions and UPD suggest that OS often has mutant or non-expressed tumor suppressor genes including two lncRNAs.
\end{abstract}

\section{Introduction}

Although it is a rare bone tumor, the genetic alterations of osteosarcoma (OS) have been studied extensively (1-5). Nevertheless, the molecular cause of OS still remains elusive. This occurs in part because the tumor is relatively rare and it is composed of a heterogeneous population of tumor cells with complex chromosomal changes $(1,2)$. OS is usually found in the metaphyseal region of the long bones especially during

Correspondence to: Dr Dechen Lin or Dr Dhong Hyun Tony Lee, Division of Hematology and Oncology, Cedars-Sinai Medical Center, UCLA School of Medicine, 8700 Beverly Blvd., Los Angeles, CA 90048, USA

E-mail: de-chen.lin@cshs.org

E-mail: dhonghyun.lee@cshs.org

Key words: osteosarcoma, copy number, uniparental disomy, lncRNAs, tumor suppressor the growth spurt in the second decade of childhood $(1,2)$. A second peak incidence of OS occurs in those who are 50 years and older (2). However, the molecular defects may be different in adults compared to children.

OS cells are generally undifferentiated $(>80 \%)$ and osteoblastic (50-80\%), suggesting that some of the initial genetic changes occur at the early stages of osteoblastogenesis $(1,2)$. In addition, many cells show a high degree of chromosomal instability (CIN) including aneuploidy ranging from haploidy to near-hexaploidy, as well as ring chromosomes, and genomic amplification as seen by homozygously staining regions (hsr), and double minutes (dmin) (1).

For better description of CIN in OS, comparative genomic hybridization $(\mathrm{CGH})$ has been widely used to date $(1,6-13)$, which confirmed the recurrent amplifications and deletions found by conventional karyotyping such as amplification at 1q21-q22, 11p14-p15, 14p11-p13, 15p11-p13, and loss of chromosome 9, 10, 13 and 17. One limitation of the technique is that it is not able to detect allele-specific chromosomal events like copy-neutral loss of heterozygosity (CN-LOH) or uniparental disomy (UPD). Acquired UPD are important chromosomal changes known to be strongly related to cancer development (14). Allelic homozygosity by LOH can lead to the selection of either inactivating mutations of tumor suppressor genes (TSG) and/or dominant negative mutations of oncogenes (14). In this study, we analyzed OS by SNP array and successfully identified many hidden regions of UPD. Our study provides valuable information that can lead to the better understanding of the molecular mechanism of OS. Importantly, our analysis led to the identification of two novel tumor suppressive lncRNAs in this malignancy.

\section{Materials and methods}

Osteosarcoma samples and extraction of genomic DNA. Fifty-eight human OS samples were obtained from several 
sources: genomic DNA and total RNA from 40 primary OS tumors and from matched normal tissues were obtained from Dr Marc Hansen, and consent forms for molecular analyses were obtained. Genomic DNA from 11 OS explants were from Dr Carl Miller, and 7 OS cell lines (U2OS, G292, MG63, HT161, HOS, SAOS2, and SJSA). The human osteoblastic cell line hFOB1.19 was used as a normal control. All OS cell lines were maintained in DMEM medium (Mediatech Inc., Herndon, VA, USA) supplemented with $10 \%$ fetal bovine serum (Atlanta Biological, Lawrenceville, GA, USA). hFOB1.19 was maintained in DMEM/F12 medium without phenol red (Invitrogen, Carlsbad, CA, USA) supplemented with 10\% FBS (Atlanta Biological) and cultured at $33^{\circ} \mathrm{C}$. All the cell lines were authenticated in April 2016, by short tandem repeat analysis with the Geneprint 10 System kit (Promega, Madison, WI, USA). Genomic DNA was isolated with DNeasy genomic DNA purification kit (Qiagen, Valencia, CA, USA) according to the manufacturer's protocol. Quality of DNA was assessed by biophotometer (Eppendorf, Hambrug, Germany) and by gel electrophoresis prior to use.

SNP array analysis. Genomic DNA from 58 human OS samples were analyzed by GeneChip Human mapping single nucleotide polymorphism (SNP) arrays (Affymetrix, Santa Clara, CA, USA) as described before $(15,16)$. 250K NspI SNP arrays were used for analysis, except for 11 explants which were analyzed by $50 \mathrm{~K} X b a \mathrm{I}$ SNP array. Briefly, $50 \mathrm{ng}$ of genomic DNA purified from each sample was digested with either $N s p$ I for $250 \mathrm{~K}$ array or with $X b a$ I for $50 \mathrm{~K}$ SNP array. Digested DNAs were ligated with adaptor and PCR amplified with LA Taq (Takara, Otsu, Shiga, Japan). Following amplification, DNA fragments were end-labeled with probes designed to differentiate SNP alleles and hybridized to SNP arrays. GeneChip Fluidics Station 400 and GeneChip scanner 3000 were used to produce raw data, which were then processed and analyzed by $\mathrm{CN}$ analyzer for Affymetrix GeneChip (CNAG 2.0) using allele-specific copy number analysis using anonymous reference (AsCNAR) algorithm (14). All allele-specific $\mathrm{CN}$ changes were automatically summarized by CNAG 2.0 using hidden-Markov model (HMM). Physical locations of chromosomal changes in the output file were based on UCSC genome browser. UPD from primary tumors with normal cell contamination were manually integrated. Copy number variants (CNV) were removed using genomic variants track in UCSC genome browser.

Validation of UPD and CN changes detected by SNP chip analysis in OS samples. Our SNP chip results were validated using genomic quantitative real-time PCR (q-PCR) and direct sequencing. Uniparental disomy (UPD) or copy-neutral loss of heterozygosity $(\mathrm{CN}-\mathrm{LOH})$ represents the loss of one allele and the duplication of the other. Therefore, a region of UPD should retain normal $\mathrm{CN}$ and have homozygosity at all SNP sites. As shown in Fig. 1A, chromosome 10 of primary tumor \#31 has normal $\mathrm{CN}$ in p-arm (10p) and UPD in q-arm (10q). To minimize the effect of nucleotide composition on the genomic q-PCR results, we chose rs10906277 and rs11199401 retaining same nucleotide composition in both normal and tumor sample (data not shown). Genomic q-PCR revealed that the $\mathrm{CN}$ of $10 \mathrm{p}$ and $10 \mathrm{q}$ regions are almost the same between the tumor and matched normal, suggesting that UPD region at $10 \mathrm{q}$ has normal CN (Fig. 1B).

Next, we checked if the UPD region showed loss of heterozygosity by nucleotide sequencing. Three independent SNP sites within the normal CN region in 10p (rs10906277, rs10828375, and rs1247098) displayed heterozygosity (Fig. 1C, upper panels) in both the tumor and matched normal sample. In contrast, 3 independent SNP sites of the UPD region in $10 \mathrm{q}$ (rs478982, rs7914572 and rs1710960) showed homozygosity (Fig. 1C, lower panels) in the tumor sample but heterozygosity in the matched normal sample, suggesting that the UPD region had $\mathrm{LOH}$ in the tumor sample. Unequal ratio of the nucleotide signals in the UPD region probably results from normal cell contamination.

For other chromosomal changes, we further extended $\mathrm{CN}$ validation by genomic q-PCR. As shown in Fig. 2A, explant \#2, \#7, \#10 and \#12 had a shared homozygous deletion of the CNTNAP2 gene at 7q35. Genomic q-PCR analysis revealed that $\mathrm{CN}$ of the CNTNAP2 gene of each of these explants were significantly lower than normal genomic DNA sample $(\mathrm{p}<0.01)$ (Fig. 2B). In contrast, five OS cell lines including SAOS2, MG63, G292 and H161 had amplification (total CN>4) and U2OS had duplication of the EXT1 gene on 8q24.11 (Fig. 2C). Genomic q-PCR of these samples showed that $\mathrm{CN}$ of the EXT1 gene was 26-fold (SAOS2), 15-fold (MG63), 12-fold (G292), 21-fold (HT161), and 2-fold (U2OS) higher than normal genomic DNA sample (Fig. 2D; $\mathrm{p}<0.01$ and $\mathrm{p}<0.05$ ). These results validated that the chromosomal changes found by SNP chip are accurate and reliable.

Plasmids and transfection. The expression plasmid of LOC285194 (pCDH-LOC285194) was kindly provided by Dr Yin-Yuan Mo (University of Mississippi Medical Center) (17). Recombinant lentiviral vectors and packaging vectors were co-transfected into $293 \mathrm{~T}$ cells using PEI transfection reagent and supernatants containing lentivirus were harvested $48 \mathrm{~h}$ after transfection as described before (18). HOS and SJSA cells were infected with the lentiviruses and supplemented with $8 \mathrm{mg} / \mathrm{ml}$ Polybrene (Sigma-Aldrich, St. Louis, MO, USA) and stable transfectants were selected with $1 \mu \mathrm{g} / \mathrm{ml}$ puromycin. For $B C 040586$, the entire sequence was cloned and inserted into pCDNA3.1 vector. The expression plasmid or the empty vector was transfected into HOS and SJSA cells using BioT reagent (Bioland Scientific LLC, Paramount, CA, USA) and stable transfectants were selected with G418 (400 ng/ml).

Cell growth analysis in vitro. Cell growth was determined by MTT (3-(4, 5-dimethylthiazol-2-yl)-2, 5-diphenyl tetrazolium bromide) assay and cell colony formation assay. In MTT assay, cells were seeded onto 96 -well plates $(2,000$ cells per well) and cell viability was assessed using MTT staining method at indicated time-points. Colony formation assay was performed by plating cells onto 6 -well plates (500 cells per well). After 2 weeks, cells were fixed with methanol and stained with crystal violet. The number of colonies was counted and data are presented as mean \pm SD from 3 independent experiments in triplicate wells.

Borden chamber cell migration assay. Cells $\left(1 \times 10^{5}\right)$ were seeded onto the top chamber of a 24-well membrane with 
A Chr 10 of Primary tumor \#31

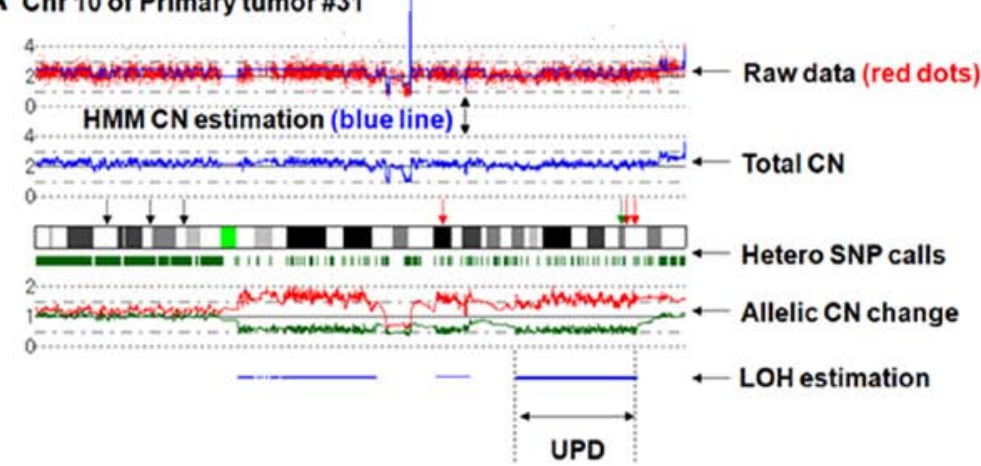

B
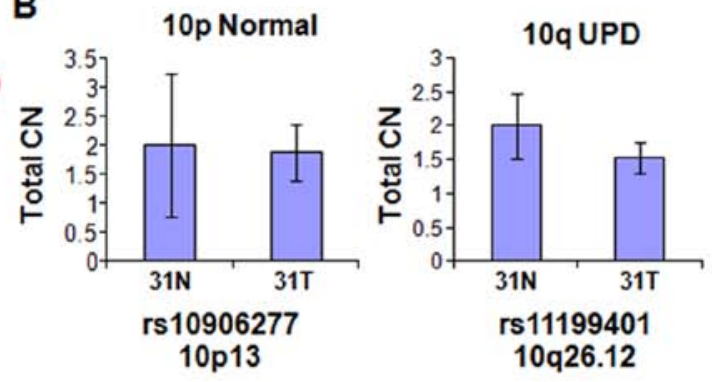
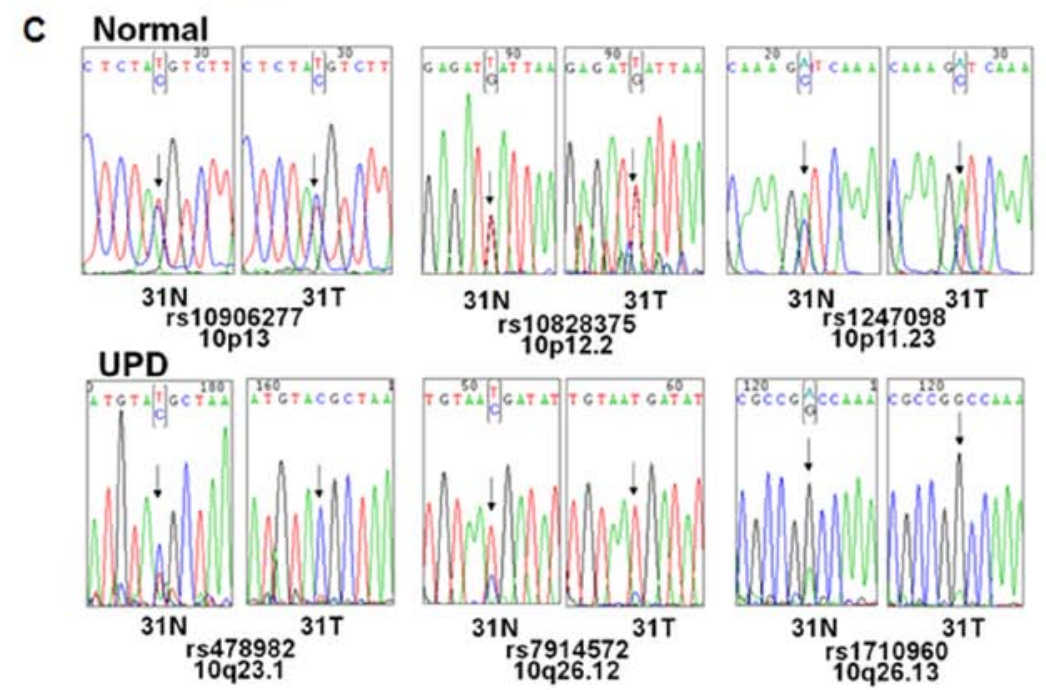

Figure 1. Overview of allele-specific copy number (CN) analysis with CNAG 2.0 and validation of UPD. (A) Overview of the SNP array data. Chromosome 13 of the primary tumor \#31 is displayed. Red dots on top are raw signals from SNPs. Total CN changes (blue lines) were estimated by the hidden-Markov model (HMM). Red and green lines at the bottom show allele-specific CN changes. Regions of loss of heterozygosity (LOH) including acquired uniparental disomy (UPD) were determined by using CNAG 2.0 software. Black arrows (rs10906277, rs10828375 and rs1247098) in the normal CN region and red arrows (rs478982, rs7914572 and rs1710960) in the UPD region indicate sequenced SNP sites. Gene dosage was measured at rs11199401 (green arrow) by genomic q-PCR. (B) Determination of total CN of the UPD region on chromosome 10 in primary tumor \#31. Total CN of the normal CN region (10p13) and UPD region (10q26.12) were measured by genomic q-PCR. Total $\mathrm{CN}$ of the normal $\mathrm{CN}$ region and the UPD region was almost the same between the tumor and the matched normal sample ( $\mathrm{p}=0.44$ and 0.88 , respectively). (C) SNP sequences on chromosome 10 in primary tumor \#31. Three independent SNP sites (rs10906277, rs10828375 and rs1247098) in the normal CN region maintained their heterozygosity in the tumor, whereas three independent SNP sites (rs478982, rs7914572 and rs1710960) in the UPD region were homozygous in the tumor sample.

8- $\mu \mathrm{m}$ pores (Thermo Fisher Scientific, Waltham, MA, USA), and the bottom chamber was filled with medium containing $10 \%$ fetal calf serum. The membranes were fixed and stained with crystal violet $24 \mathrm{~h}$ after seeding, and migrated cells were quantified by microscopically counting 5 random fields at a magnification of x200. Mean values were calculated from data obtained from three separate chambers.

Wound-healing assay. In wound-healing assays, cells were plated and grown to confluence for $24 \mathrm{~h}$, then cells were starved in serum-free medium for $12 \mathrm{~h}$, and a scratch was made across the monolayer using a sterile pipette tip. Wound closure result was imaged at 0 and $24 \mathrm{~h}$ with a microscope (x200).

Tumorigenesis experiment in nude mice. In vivo tumorigenesis assay was performed as described previously (19). In brief, ten 6-week-old female nude mice were subcutaneously injected with $2 \times 10^{6}$ HOS cells (overexpression of indicated lncRNA) on their dorsal flanks, with each mouse carrying four explants. Tumor growth was monitored and tumor size was measured every 3 days. Tumor volume was calculated using the formula, volume $=1 / 2\left(\right.$ length $\mathrm{x}$ width $\left.^{2}\right)$. After 20 days, the mice were sacrificed and the tumors were removed and weighed. All animal studies were conducted in accordance with NIH animal use guidelines and were approved by the Institutional Animal Care and Use Committee (IACUC) at Cedars-Sinai Medical Center.

Statistical analysis. Two-tailed Student's t-test was used to analyze the potential statistical difference between two groups. Statistical significance was set at $\mathrm{p}<0.05$.

\section{Results}

Recurrent UPD regions in $O S$. Among thousands of recognizable chromosomal changes in 59 OS samples, we found $950 \mathrm{LOH}$ regions including 355 sites of UPD (data available upon request). All samples had LOH. To our surprise, 37\% of LOH were in the form of UPD, highlighting its prevalence in OS. Interestingly, a total of $98 \mathrm{LOH}$ events spanned the 
A $\operatorname{Chr} 7$

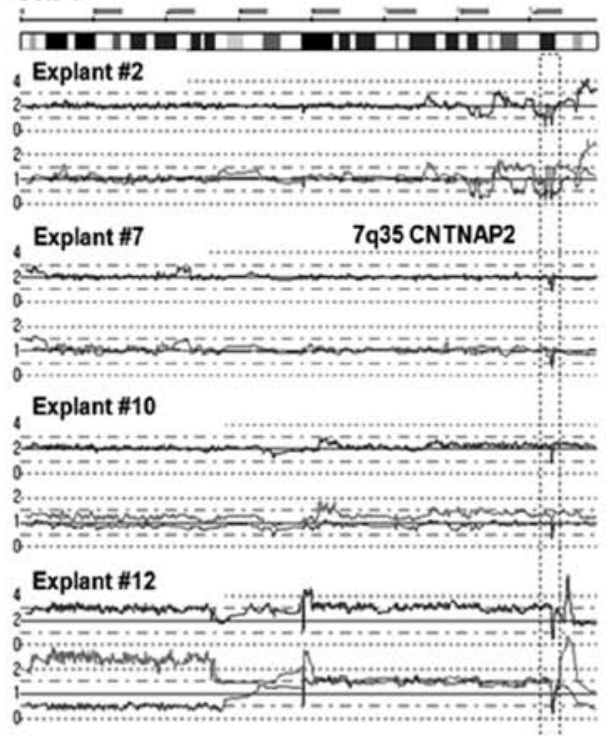

B

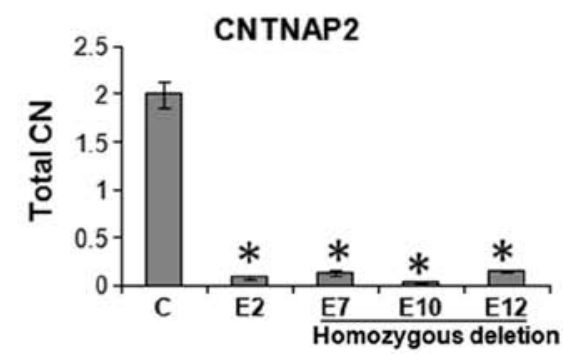

C $\mathrm{Chr} 8$
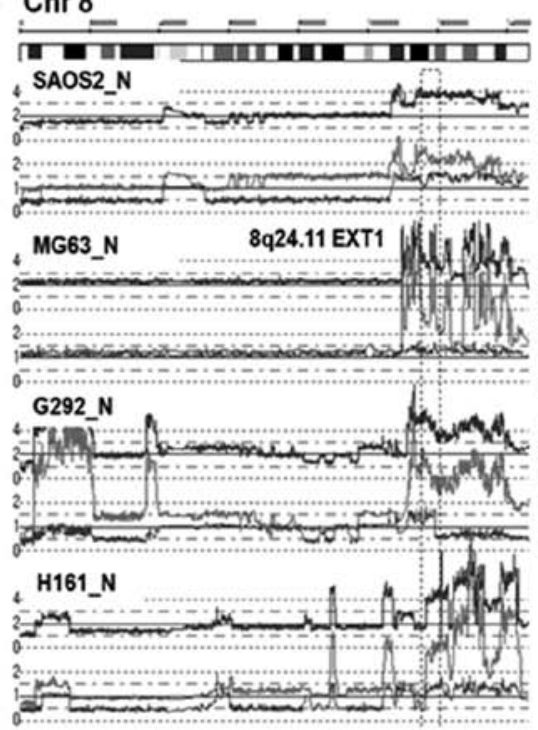

Chr 8
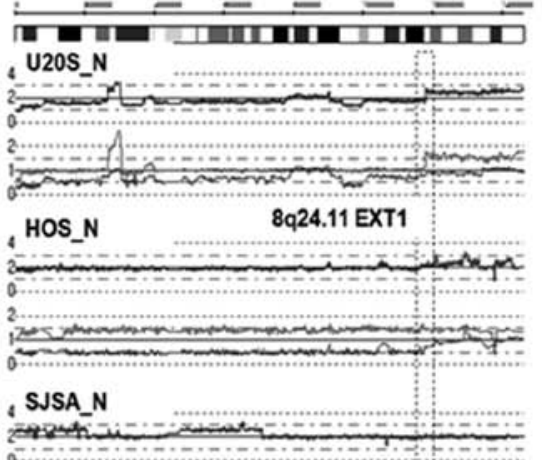
and

D

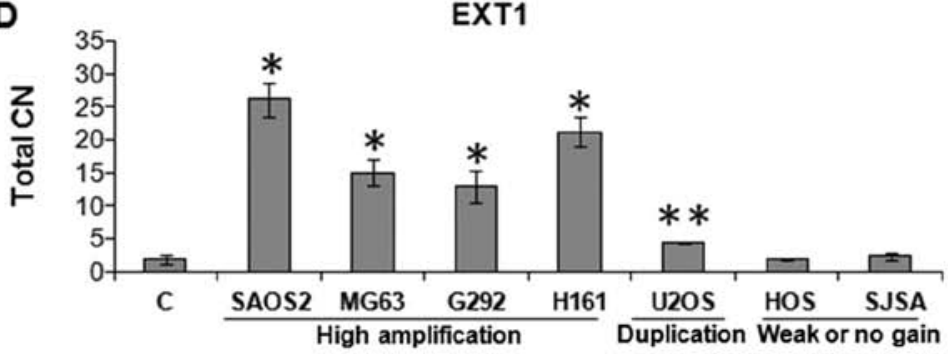

Figure 2. Validation of total CN changes. (A) SNP array results of explant \#2, \#7, \#10 and \#12. Each has a homozygous deletion (HD) of the internal region of CNTNAP2 gene at 7q35. (B) Total CN of the 7q35 HD region in explants \#2, \#7, \#18 and \#12 were 10-fold lower than normal genomic DNA sample ( $\mathrm{C}$ indicates normal genomic DNA control. Primer pair specific for the HD region was used for quantitation. (C) SNP array results of 7 OS cell lines on chromosome 8. OS cell lines, SAOS2, MG63, G292 and HT161 showed high amplification; U2OS and HOS had either duplication or weak allelic gain, respectively; and SJSA showed normal CN at 8q23.3-q24 region. (D) Total CN of the 8q24.11 region was 26-fold (SAOS2), 15-fold (MG63), 12-fold (G292) and 21-fold (HT161) higher than that of normal genomic DNA ( $\left.{ }^{* *} \mathrm{p}<0.01\right)$. U2OS showed 2 -fold increase ( $\left.\mathrm{p}<0.05\right)$. HOS and SJSA cells have almost same levels compared to normal genomic DNA. Primer pair specific for EXT1 in 8q24.11 was used for quantitation.

whole chromosome, among which, $53 \mathrm{LOH}$ were monosomy and $45 \mathrm{LOH}$ were whole-chromosomal UPD. Monosomy occurred most frequently at chromosomes 13 and 16 (7 cases each), no monosomy was identified at chromosomes 4,5 and 7. Whole-chromosomal UPD (Fig. 3A) was most frequent at chromosome 22 ( 5 cases) followed by chromosomes 2,13 and 18 (4 cases each). No whole-chromosomal UPD was noted at either chromosome 1,8,14 or 17. Interestingly, chromosomes $1,8,14$ and 17 had regions of recurrent high amplification (discussed later).

Concerning segmental UPD (Figs. 1C and 3B), chromosome 12 q-arm (12q) UPD occurred the most frequently in 13 samples (22\%). The next most common was 17q UPD found in 12 samples (20\%); by incidence, 12q UPD was also the most frequent (18 regions) followed by 13q UPD (16 regions).

Seventy percent of the primary samples had one or more recurrent UPD regions, and $100 \%$ of cell lines and explants had recurrent UPD regions. Regions of recurrent UPD appeared in various sizes and chromosomal loci (Table I). Excluding whole-chromosomal UPD, the median size of the UPD regions was $11 \mathrm{Mb}$, ranging from $2 \mathrm{Mb}$ (Xp22.12 and Xp22.12-p21.3) to $54 \mathrm{Mb}$ (whole q-arm UPD of chromosome 16).
Moreover, recurrent UPD regions contained genes known to be frequently mutated in OS (Table I). For example, $R B I$ and TP53, two of the most commonly mutated genes in OS $(1,2)$, occurred in the recurrent UPD regions of chromosomes 13 and 17, respectively. $C D K N 2 A$ and $C D K N 2 B$, two well-known TSG, were not only in a recurrent UPD region, but also showed recurrent homozygous deletions (HD) in many samples (Table II). EGFR gene was also found in the recurrent UPD region of chromosome 7 . We have extensively validated UPD and $\mathrm{CN}$ changes (shown below) through both genomic q-PCR and direct sequencing (Materials and methods and Figs. 1 and 2).

Recurrent amplifications and homozygous deletions in OS. We analyzed total $\mathrm{CN}$ changes and identified common regions of amplification showing $\mathrm{CN}$ estimation of 5 and 6 determined by HMM. Eleven regions were commonly amplified region in OS cells (Table III). Common amplifications at 1q21.1-1q24.1, 6p21.1-p12.1, 8q23.3-q24.3, 14q11.2-q12 and 17p12-p11 have been reported (1-13), showing that these region are frequently amplified in OS cells. Next, we identified homozygous deletions (HD) and found 196 recurrent HD in total (Table II). 
Table I. Recurrent UPD regions in OS.

\begin{tabular}{|c|c|c|c|c|c|c|}
\hline \multirow[b]{2}{*}{ Cytoband } & \multicolumn{2}{|c|}{ UCSC May 2004 assembly } & \multirow[b]{2}{*}{ Size $(\mathrm{Mb})$} & \multicolumn{2}{|c|}{ Samples } & \multirow[b]{2}{*}{ Known mutated gene in $\mathrm{OS}^{\mathrm{a}}$} \\
\hline & StartPOS & EndPOS & & Total no. & Total $\%$ & \\
\hline 1q31.1-q41 & 183188858 & 211490840 & 28 & 10 & 17 & \\
\hline 2p24.1-p12 & 19495270 & 76950296 & 57 & 10 & 17 & \\
\hline $2 q 24.2-q 33.1$ & 160740613 & 199983101 & 39 & 9 & 16 & \\
\hline $3 \mathrm{p} 22.3-\mathrm{p} 14.2$ & 36726626 & 60749157 & 24 & 12 & 21 & \\
\hline $3 q 11.2-q 13.31$ & 98705120 & 117729514 & 19 & 8 & 14 & \\
\hline 4q34.1-qter & 172743172 & 191290799 & 19 & 8 & 14 & \\
\hline $5 q 11.2-q 14.1$ & 55246197 & 80739073 & 25 & 6 & 10 & \\
\hline $5 q 22.1-q 31.3$ & 109913296 & 141494150 & 32 & 6 & 10 & \\
\hline $6 q 13-q 14.1$ & 72226093 & 79985449 & 8 & 9 & 16 & \\
\hline $6 q 22.31-q 24.2$ & 121318426 & 143982816 & 23 & 9 & 16 & \\
\hline 7p12.3-p11.1 & 47268854 & 57187722 & 10 & 9 & 16 & $\operatorname{EGFR}\left(6 \%{ }^{\mathrm{b}}\right)$ \\
\hline 8p21.3-p12 & 21242912 & 30905027 & 10 & 4 & 7 & \\
\hline $8 \mathrm{q} 22.1-\mathrm{q} 22.3$ & 96630283 & 104495421 & 8 & 4 & 7 & \\
\hline 9p21.3-p12 & 22923651 & 44108554 & 21 & 6 & 10 & $C D K N 2 A, 2 B(11 \%)$ \\
\hline 9q33.1-qter & 115491394 & 135945601 & 20 & 7 & 12 & \\
\hline 10q11.21-q22.1 & 44497840 & 70380364 & 26 & 9 & 16 & \\
\hline $10 \mathrm{q} 25.2-\mathrm{q} 26.12$ & 114228361 & 122214789 & 8 & 8 & 14 & \\
\hline 11pter-p13.1 & 1938894 & 30198901 & 28 & 8 & 14 & \\
\hline $11 q 22.3-q 23.3$ & 103485551 & 114473112 & 11 & 8 & 14 & \\
\hline 11q24.2-qter & 124730067 & 134437775 & 10 & 8 & 14 & \\
\hline 12 p13.2-p12.3 & 11980008 & 15490246 & 4 & 9 & 16 & \\
\hline $12 q 11-q 12$ & 36391876 & 43990452 & 8 & 9 & 16 & \\
\hline $12 \mathrm{q} 24.31-\mathrm{q} 24.32$ & 123828884 & 128205825 & 4 & 12 & 21 & \\
\hline $13 q 11-q 12.3$ & 18209780 & 28993301 & 11 & 9 & 16 & \\
\hline $13 q 14.11-q 21.1$ & 39159522 & 56979282 & 18 & 10 & 17 & $R B 1(13 \%)$ \\
\hline $14 q 21.3-q 24.1$ & 48497095 & 57242510 & 9 & 3 & 5 & \\
\hline $15 q 21.3-q 22.31$ & 56990907 & 64239300 & 7 & 9 & 16 & \\
\hline 16pter-p12.1 & 205160 & 22749097 & 23 & 9 & 16 & \\
\hline 16q11.2-qter & 45226833 & 88666241 & 43 & 12 & 21 & \\
\hline 17p13.2-p12 & 5999210 & 11680056 & 6 & 12 & 21 & TP53 (30\%) \\
\hline $18 \mathrm{p} 11.22-\mathrm{p} 11.21$ & 8617957 & 15073063 & 6 & 8 & 14 & \\
\hline $18 \mathrm{q} 11.2-\mathrm{q} 12.1$ & 20243361 & 25176204 & 5 & 8 & 14 & \\
\hline 19p13.2-p13.13 & 6909134 & 13445855 & 7 & 7 & 12 & \\
\hline $19 q 13.11-q 13.42$ & 40074074 & 58908108 & 19 & 6 & 10 & \\
\hline 20q11.21-q13.12 & 30491788 & 41744225 & 11 & 7 & 12 & \\
\hline 20q13.2-q13.33 & 49983933 & 60249150 & 10 & 6 & 10 & \\
\hline 21q22.12-qter & 34975513 & 46885639 & 12 & 6 & 10 & \\
\hline $22 q 12.3$ & 31598720 & 33627072 & 2 & 10 & 17 & \\
\hline 22q12.3-q13.1 & 35390989 & 37641604 & 2 & 10 & 17 & \\
\hline Xp22.12-p21.3 & 20242329 & 27726023 & 7 & 4 & 7 & \\
\hline Xq27.2-qter & 141498847 & 154353200 & 13 & 5 & 9 & \\
\hline
\end{tabular}

${ }^{\mathrm{a}}$ Genes known to be mutated in OS as determined by COSMIC database (release 37). ${ }^{\mathrm{b}}$ Percentage in parenthesis indicates the mutation rate determined by COSMIC database. StartPOS, start base position; EndPOS, end base position.

Many HD occurred in the vicinity of genes located at common fragile sites (CFS) e.g., LRP1B (FRA2F), CNTNAP2 (FRA7I), $M A G I 2$ (FRA7E), DLG2 (FRA11F) and DMD (FRAXC). These genes are deleted in many cancers.
Importantly, homozygous deletion of $3 q 13.31$ was the most frequent HD (10 cases; $17 \%$ of the samples) in our cohort. This region contained either a 5'-portion of LSAMP gene, lncRNAs LOC285194 or BC040587, or all three, in agreement with 

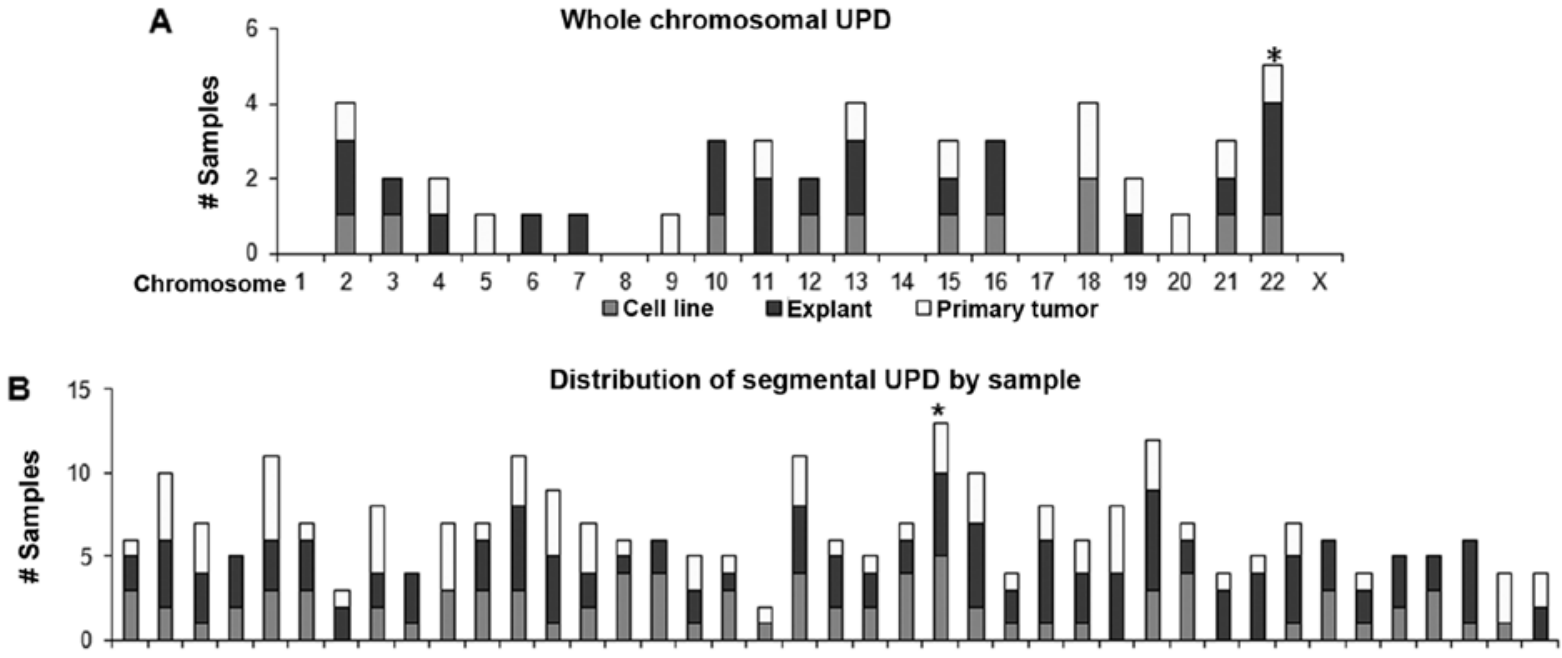

Chromosome $\frac{p q}{1} \frac{p q}{2} \frac{p q}{3} \frac{p q}{4} \frac{p q q}{5} \frac{p q}{6} \frac{p q}{7} \frac{p q q}{8} \frac{p q}{9} \frac{p q}{10} \frac{p q}{11} \frac{p q q}{12} \frac{q}{13} \frac{q}{14} \frac{p q}{16} \frac{p q}{17} \frac{p q}{18} \frac{p q}{19} \frac{p q q}{20} \frac{q}{21} \frac{p q}{x}$ 口Cell line axplant aPrimary tumor

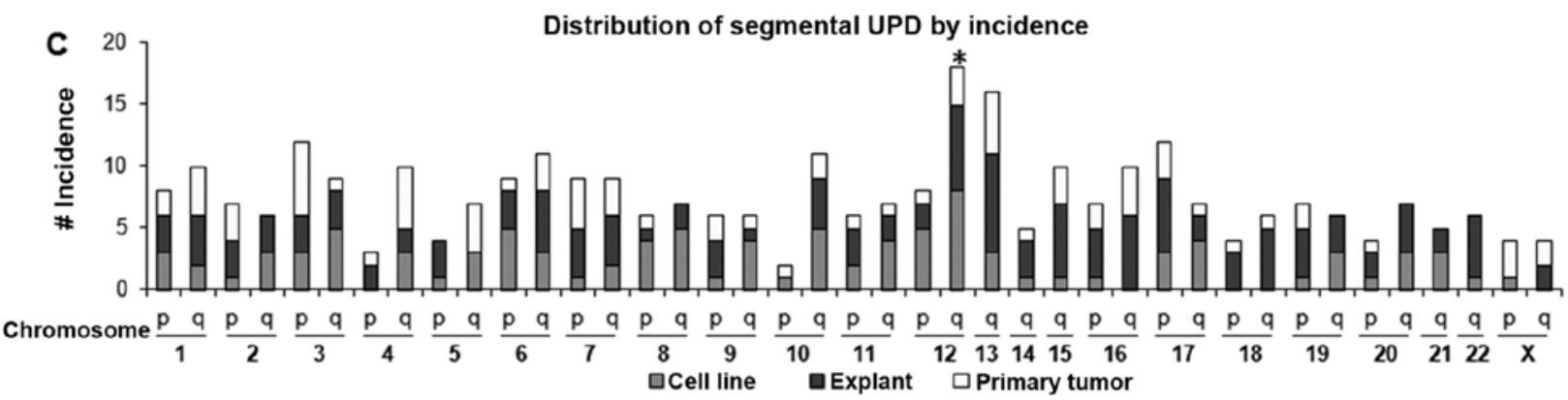

Figure 3. Chromosomal distribution of UPDs in OS samples. All chromosomes in OS had a certain number of UPDs. Blue, purple and yellow bars represent the number of UPDs in cell lines, explants and primary tumors, respectively. The highest peak of each graph is marked with an asterisk (*). (A) Distribution of whole-chromosomal UPD. (B) Distribution of segmental UPD. Samples with more than one UPD region were counted as one. (C) Distribution of segmental UPD by incidence. Whole-chromosomal UPD was not counted.

Table II. Recurrent homozygous deletions in OS.

\begin{tabular}{|c|c|c|c|c|c|c|}
\hline \multirow[b]{2}{*}{ Cytoband } & \multicolumn{2}{|c|}{ UCSC May 2004 assembly } & \multirow[b]{2}{*}{ Size $(\mathrm{Mb})$} & \multicolumn{2}{|c|}{ Samples } & \multirow[b]{2}{*}{$\begin{array}{l}\text { Genes and miRNAs }{ }^{\mathrm{a}} \text { within } \\
\text { the region }\end{array}$} \\
\hline & StartPOS & EndPOS & & Total no. & Total \% & \\
\hline $2 q 22.1$ & 141810352 & 141928225 & 0.1 & 3 & 5 & $L R P 1 B$ \\
\hline $3 \mathrm{p} 13$ & 71522662 & 77688970 & 6.1 & 3 & 5 & $F O X P 1, R O B O 2$ \\
\hline $3 q 13.31$ & 117074192 & 118436759 & 9.2 & 9 & 16 & LSAMP, LOC285194 BC040587 \\
\hline $7 q 21.11$ & 77389932 & 77976116 & 0.6 & 2 & 3 & $M A G I 2$ \\
\hline $7 q 35-q 36.3$ & 146128574 & 146341779 & 0.2 & 5 & 9 & CNTNAP2 \\
\hline $9 \mathrm{p} 21.3$ & 21801533 & 22203270 & 0.4 & 4 & 7 & $C D K N 2 A, 2 B$ \\
\hline $11 q 14.1$ & 82617545 & 84343329 & 1.7 & 2 & 3 & $D L G 2$ \\
\hline $13 q 14.2$ & 47914064 & 47963566 & 0.05 & 2 & 3 & $R B 1$ \\
\hline $13 q 14.2$ & 48209946 & 49643972 & 1.4 & 3 & 5 & DLEU1, mir15a, mirl6-1 \\
\hline Xp22.33 & 3491918 & 3532609 & 4.1 & 4 & 7 & $P R K X$ \\
\hline $\mathrm{Xp} 21.1$ & 31891496 & 33362759 & 1.5 & 4 & 7 & $D M D$ \\
\hline
\end{tabular}

${ }^{\mathrm{a} G e n e s ~ l i s t e d ~ a r e ~ t h e ~ o n l y ~ g e n e ~ o r ~ g e n e s ~ i n ~ t h e ~ r e g i o n . ~ S t a r t P O S, ~ s t a r t ~ b a s e ~ p o s i t i o n ; ~ E n d P O S, ~ e n d ~ b a s e ~ p o s i t i o n . ~}$

a recent report (20). In many cases, deletion of LOC285194 and $B C 040587$ ( 7 cases) was more frequent than deletion of
LSAMP gene (3 cases), suggesting that these two lncRNAs might be biologically relevant in OS cells. 
Table III. Recurrent high amplifications in OS.

\begin{tabular}{|c|c|c|c|c|c|c|}
\hline \multirow[b]{2}{*}{ Cytoband } & \multicolumn{2}{|c|}{ UCSC May 2004 assembly } & \multirow[b]{2}{*}{ Size $(\mathrm{Mb})$} & \multicolumn{2}{|c|}{ Samples } & \multirow[b]{2}{*}{ Known oncogenes } \\
\hline & StartPOS & EndPOS & & Total no. & Total \% & \\
\hline 1p31.2-p21.1 & 68271671 & 102822954 & 35 & 5 & 9 & \\
\hline $1 q 21.1-q 24.1$ & 142406039 & 163411899 & 21 & 8 & 14 & \\
\hline $4 q 11-q 12$ & 52525819 & 65971362 & 13 & 6 & 10 & $K I T$ \\
\hline $5 \mathrm{p} 13.1-\mathrm{q} 11.2$ & 38854038 & 50857095 & 12 & 6 & 10 & \\
\hline 6p21.1-p12.1 & 44140309 & 53601419 & 9 & 8 & 14 & \\
\hline $8 q 23.3-q 24.3$ & 113293800 & 141257873 & 28 & 9 & 16 & $M Y C$ \\
\hline $14 q 11.2-q 12$ & 19285288 & 30330681 & 11 & 10 & 17 & \\
\hline 17p12-p11.2 & 12859705 & 22691152 & 10 & 9 & 16 & $C R K$ \\
\hline $17 q 23.2-q 25.3$ & 56600056 & 75827015 & 19 & 6 & 10 & \\
\hline $19 q 12$ & 32842516 & 36885229 & 4 & 8 & 14 & \\
\hline Xp11.1-q12 & 57426758 & 65696889 & 8 & 5 & 9 & \\
\hline
\end{tabular}

The high amplifications have $>4$ copies determined by hidden-Markov model (HMM). StartPOS, start base position; EndPOS, end base position.
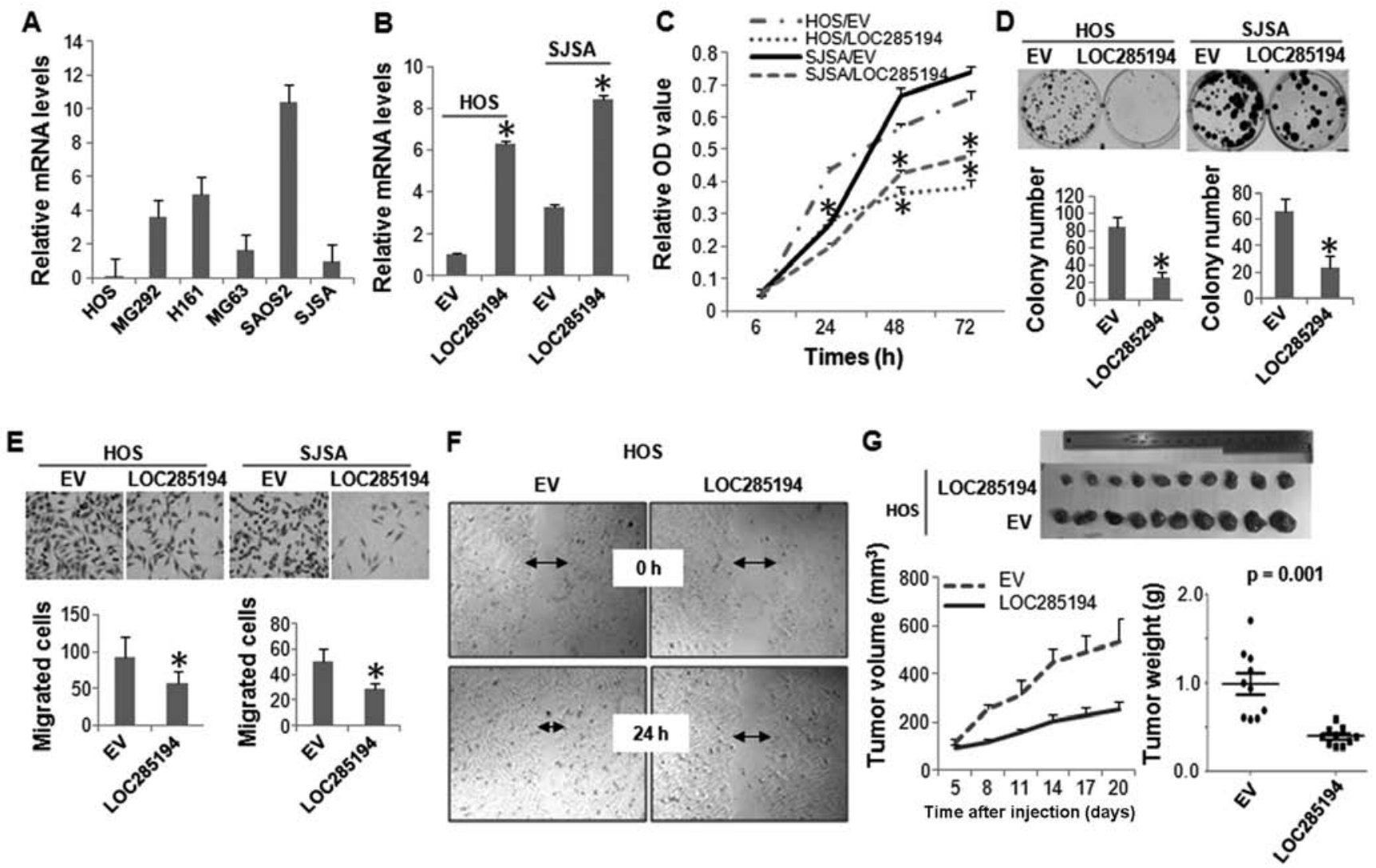

Figure 4. Effect of LOC285194 overexpression on the growth and migration of OS cells. (A) qRT-PCR measured mRNA levels of LOC285194 in a panel of OS cell lines. (B) Expression plasmids encoding either LOC285194 (LOC285194) or empty vector (EV) were transfected into HOS cells and SJSA OS cells and expression of Loc285194 was analyzed by qRT-PCR. (C) Growth curve of these cells was determined using MTT assay. (D) Colony formation assay measuring the effect of Loc285194 restoration on cell proliferation. (E and F) Boyden chamber migration assay (E) and wound-healing assay (F) evaluating cell migration. Images of representative migrated tumor cells (x200); data represent mean \pm SD of triplicates. (G) HOS cells with either ectopic expression of stable LOC285194 or empty vector were implanted subcutaneously into nude mice. Tumor volume at different time-points after implantation (lower left panel) and average weight of the tumors at termination of the experiment (lower right panel) were analyzed. ${ }^{*} \mathrm{p}<0.05$.

Both LOC285194 and BC040587 play important roles in the proliferation and migration of OS cells. Although LOC285194 has been implicated as a tumor suppressor lncRNA in OS (20), its function has not been studied extensively. Moreover, the 


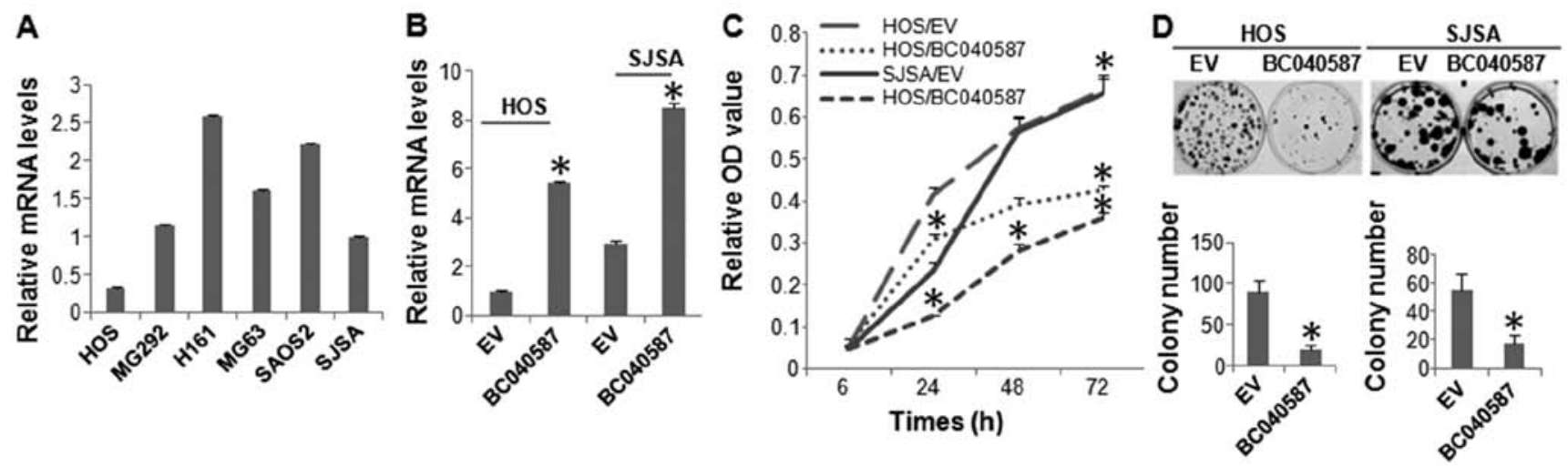

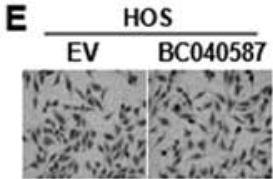
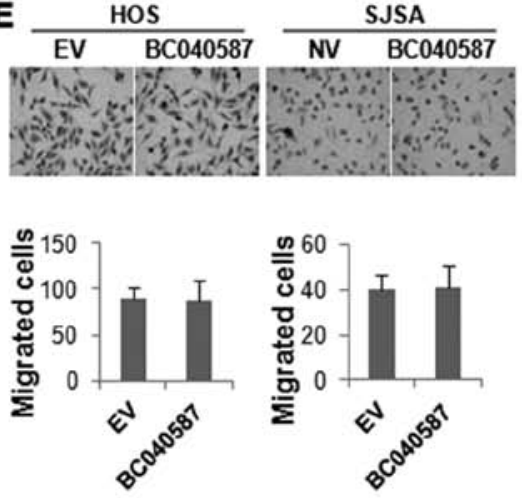

$\mathbf{F}$

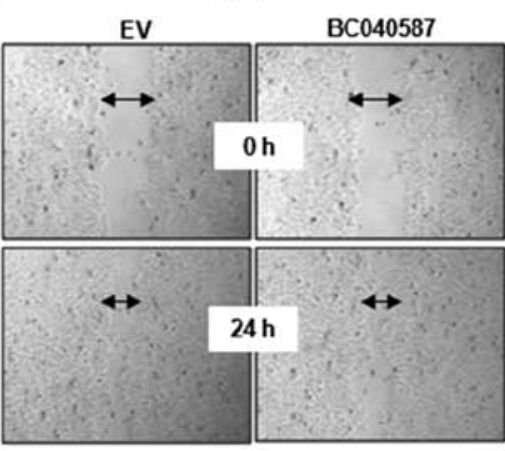

G
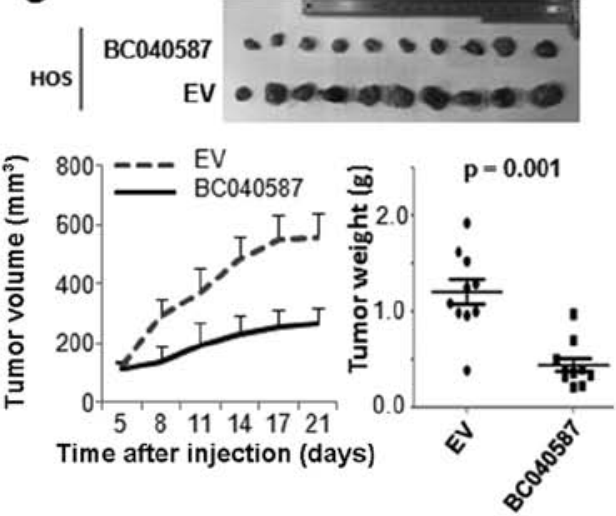

Figure 5. Roles of BC040587 on growth and migration of OS cells. (A) The mRNA levels of BC040587 in OS cell lines were measured by qRT-PCR. (B) BC040587 was ectopically expressed in HOS cells and SJSA cells. (C) MTT assay and (D) colony formation assay measured the proliferation of OS cells expressing either BC040587 (BC040587) or empty vector control (EV). (E and F) Boyden chamber migration assay (E) and wound healing assay (F) evaluating cell migration. (G) Stable BC040587-expressing HOS cells were inoculated into nude mice and tumor volume (lower left panel) and tumor weight (lower right panel) were recorded. "p $<0.05$.

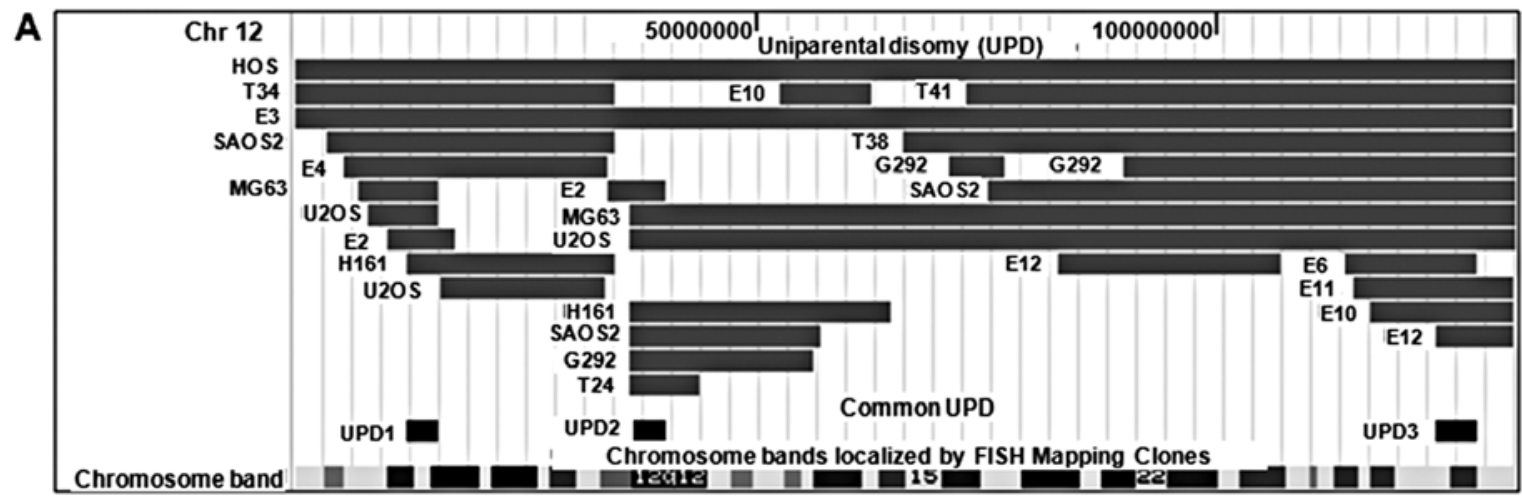

B

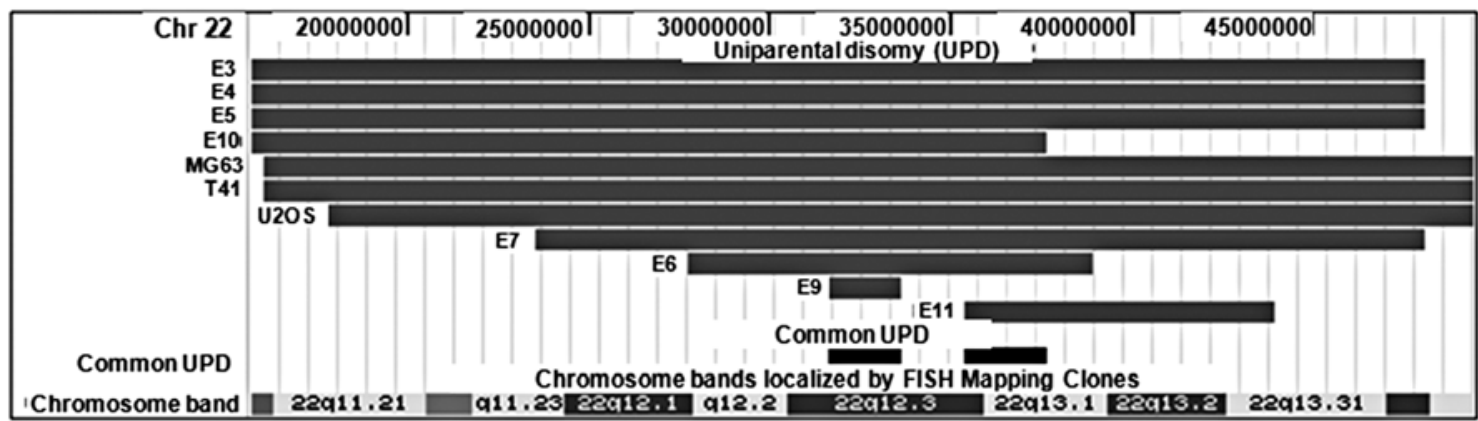

Figure 6. Recurrent UPD regions in chromosomes 12 and 22. (A) Summary of chromosome 12 UPD. Three distinct recurrent UPD regions were identified: UPD1 at 12p13.31-p12.3, UPD2 at 12q11-q12, and UPD3 at 12q24.31-qter. (B) Summary of chromosome 22 UPD. Two recurrent UPD regions were identified: UPD1 at 22q12.3 and UPD2 at 22q12.3-q23.1. 
biological relevance of $\mathrm{BC} 040587$ remains unknown in the context of OS. To address this, we next sought to characterize the functional roles of both LOC285194 and BC040587 in OS cells.

Expression level of LOC285194 was determined in a panel of OS cell lines by qRT-PCR analysis. It was expressed at low levels in all the OS cells lines evaluated. Not surprisingly, LOC285194 was almost undetectable in HOS cells, which has HD at this genomic locus (Fig. 4A). We next ectopically expressed LOC285194 in both HOS and SJSA cells, both displayed the lowest expression of this lncRNA (Fig. 4B). Both MTT and colony formation assays showed that restoration of LOC285194 markedly attenuated the proliferation of both HOS and SJSA cells (Fig. 4C and D). Moreover, Boyden chamber migration assay and wound-healing assay revealed that LOC285194 expression resulted in a significantly decreased cell migration (Fig. 4E and F). To determine whether LOC285194 regulates xenograft growth of OS cells in vivo, we injected either LOC285194-restored or control HOS cells into the nude mice. Consistent with the effects of LOC285194 expression in vitro, LOC285194 potently inhibited xenograft growth (Fig. 4G).

We next performed biological assays to determine the functional role of the other lncRNA, BC040587, in OS cells. Similar to LOC285194, HOS cells expressed the lowest level of BC040587, followed by SJSA cells (Fig. 5A). BC040587 was ectopically expressed in these two cell lines (Fig. 5B), which markedly inhibited their cellular proliferation (Fig. 5C and D). However, unlike LOC285194, expression of BC040587 did not affect the migration of the OS cells (Fig. 5E and F). The tumor suppressive function of BC040587 was further confirmed by in vivo xenograft assay (Fig. $5 \mathrm{G}$ ).

\section{Discussion}

Unlike CGH studies which focused on primary tumors, our study used OS from three different sources: 7 cell lines, 11 explants and 40 primary tumors. The cell lines and explants maintained the chromosomal aberrations found in primary tumors. The same phenomenon was observed in malignant melanoma where the overall genomic profiles of 101 melanoma cell lines nearly matched those of 70 primary melanomas (21). Similar concordness was noted between 26 pancreatic cancer cell lines and primary pancreatic cancers (22). Thus, combining the results of all 59 samples into one analysis of common chromosomal changes in OS is reasonable.

Our analysis particularly focused on the study of $\mathrm{LOH}$, especially UPD. Notably, almost $37 \%$ of the LOH were hidden as UPD. Although surprising, esophageal cancer (23) and basal cell carcinoma (24) also had UPD, with $33 \%$ of the total number of chromosomal changes and $42 \%$ of the total $\mathrm{LOH}$, respectively.

Among all UPD regions, 12q UPD occurred with the highest frequency (18 events) affecting the highest number of samples (13 samples, 23\%). Two recurrent UPD regions were identified on 12q: 12q11-q12 (UPD2) and 12q24.31-q24.32 (UPD3) (Fig. 6A). Interestingly, the location of the recurrent UPD regions, either centromeric (UPD2) or telomeric (UPD3), suggests that these UPD regions were a result of somatic recombination.
Although its incidence was relatively lower than chromosome 12 , chromosome 22 UPD was interesting in that nearly half of the UPD (45\%) (Fig. 6B), involved the whole-chromosome suggesting that the mechanism of UPD formation might be different from those of other UPD regions. Among the genes in the region, $C S N K 1 E$ gene is a potential target because of its important role in canonical Wnt signaling pathway and in circadian rhythm signaling pathway. Interestingly, many genes in the recurrent UPD regions play major roles in the Wnt signaling pathway, especially in the canonical Wnt signaling pathway (data not shown). Previous research shows that canonical Wnt signaling pathway is deregulated in OS $(25,26)$. For example, $\mathrm{Ng}$ et al (27) checked the cellular location of $\beta$-catenin in 545 mesenchymal tumors including 19 OS and found that OS showed an accumulation of $\beta$-catenin in nucleus and/or in cytoplasm.

Frequent HD involving 3q13.31 region has been observed in OS $(20,28,29)$. Among the genes located within the deleted region, $L S A M P$, together with two IncRNAs LOC285194 and $B C 040587$, were highlighted. Variable levels of these two lncRNAs in different osteosarcoma cell lines were observed. It is not surprising that LOC285194 and BC040587 were expressed at their lowest level in HOS cells, as this line has a homozygous deletion at this genomic locus. Among the other cell lines, no appreciable copy number changes were detected, and we reasoned that the different RNA levels might result from epigenetic alterations, such as DNA methylation or histone modification. Previous reports noted that silencing LOC285194 promoted proliferation of normal osteoblasts, and HD of LOC285194 or BC040587 was associated with a poor survival of OS patients $(17,20)$. We show that restoration of either LOC285194 or BC040587 in OS cell lines potently suppressed cell proliferation in vitro and in vivo, consistent with the tumor suppressive roles of these two lncRNAs. We also found that expression of LOC285194 but not BC040587 inhibited the migration of OS cells. Continuing research will be needed to elucidate the mode of action of these two lncRNAs.

In conclusion, we discovered that OS has frequent deletions and many of these represent chromosome region with mutation of one allele of a target gene and its duplication with less of the second normal allele. These target genes are usually tumor suppressor genes. Hence, OS probably has numerous mutant tumor suppressor genes.

\section{Acknowledgements}

This study was supported by the Singapore Ministry of Health's National Medical Research Council (NMRC) under its Singapore Translational Research (STaR) Investigator Award to H.P.K., NMRC Individual Research Grant (NMRC/1311/2011) and the NMRC Centre Grant awarded to National University Cancer Institute of Singapore, the National Research Foundation Singapore and the Singapore Ministry of Education under its Research Centres of Excellence initiatives to H.P.K. D.-C.L. was supported by American Society of Hematology Fellow Scholar Award, Donna and Jesse Garber Awards for Cancer Research and National Center for Advancing Translational Sciences UCLA CTSI Grant UL1TR000124. This study was also supported by the Wendy Walk Foundation, Slifka Foundation and the RNA Biology 
Center at the Cancer Science Institute of Singapore, NUS, as part of funding under the Singapore Ministry of Education's Tier 3 grants (grant no. MOE2014-T3-1-006).

\section{References}

1. Sandberg AA and Bridge JA: Updates on the cytogenetics and molecular genetics of bone and soft tissue tumors: Osteosarcoma and related tumors. Cancer Genet Cytogenet 145: 1-30, 2003.

2. Kansara M and Thomas DM: Molecular pathogenesis of osteosarcoma. DNA Cell Biol 26: 1-18, 2007.

3. Savage SA, Mirabello L, Wang Z, Gastier-Foster JM, Gorlick R, Khanna C, Flanagan AM, Tirabosco R, Andrulis IL, Wunder JS, et al: Genome-wide association study identifies two susceptibility loci for osteosarcoma. Nat Genet 45: 799-803, 2013.

4. Mirabello L, Koster R, Moriarity BS, Spector LG, Meltzer PS, Gary J, Machiela MJ,Pankratz N, Panagiotou OA, LargaespadaD, et al: A genome-wide scan identifies variants in NFIB associated with metastasis in patients with osteosarcoma. Cancer Discov 5: 920-931, 2015

5. Kovac M, Blattmann C, Ribi S, Smida J, Mueller NS, Engert F, Castro-Giner F, Weischenfeldt J, Kovacova M, Krieg A, et al: Exome sequencing of osteosarcoma reveals mutation signatures reminiscent of BRCA deficiency. Nat Commun 6: 8940, 2015.

6. dos Santos Aguiar S, de Jesus Girotto Zambaldi L, dos Santos AM, Pinto W Jr and Brandalise SR: Comparative genomic hybridization analysis of abnormalities in chromosome 21 in childhood osteosarcoma. Cancer Genet Cytogenet 175: 35-40, 2007.

7. Ohata N, Ito S, Yoshida A, Kunisada T, Numoto K, Jitsumori Y, Kanzaki H, Ozaki T, Shimizu K and Ouchida M: Highly frequent allelic loss of chromosome 6q16-23 in osteosarcoma: Involvement of cyclin C in osteosarcoma. Int J Mol Med 18: $1153-1158,2006$

8. Atiye J, Wolf M, Kaur S, Monni O, Böhling T, Kivioja A, Tas E, Serra M, Tarkkanen M and Knuutila S: Gene amplifications in osteosarcoma-CGH microarray analysis. Genes Chromosomes Cancer 42: 158-163, 2005.

9. Zielenska M, Marrano P, Thorner P, Pei J, Beheshti B, Ho M, Bayani J, Liu Y, Sun BC, Squire JA, et al: High-resolution cDNA microarray CGH mapping of genomic imbalances in osteosarcoma using formalin-fixed paraffin-embedded tissue. Cytogenet Genome Res 107: 77-82, 2004.

10. Man TK, Lu XY, Jaeweon K, Perlaky L, Harris CP, Shah S, Ladanyi M, Gorlick R, Lau CC and Rao PH: Genome-wide array comparative genomic hybridization analysis reveals distinct amplifications in osteosarcoma. BMC Cancer 4: 45, 2004.

11. Lau CC, Harris CP, Lu XY, Perlaky L, Gogineni S, Chintagumpala M, Hicks J, Johnson ME, Davino NA, Huvos AG, et al: Frequent amplification and rearrangement of chromosomal bands 6 p12-p21 and 17 p11.2 in osteosarcoma. Genes Chromosomes Cancer 39: 11-21, 2004.

12. Squire JA, Pei J, Marrano P, Beheshti B, Bayani J, Lim G, Moldovan L and Zielenska M: High-resolution mapping of amplifications and deletions in pediatric osteosarcoma by use of CGH analysis of cDNA microarrays. Genes Chromosomes Cancer 38: 215-225, 2003.

13. Overholtzer M, Rao PH, Favis R, Lu XY, Elowitz MB, Barany F, Ladanyi M, Gorlick R and Levine AJ: The presence of p53 mutations in human osteosarcomas correlates with high levels of genomic instability. Proc Natl Acad Sci USA 100: 11547-11552, 2003.

14. Yamamoto G, Nannya Y, Kato M, Sanada M, Levine RL, Kawamata N, Hangaishi A, Kurokawa M, Chiba S, Gilliland DG, et al: Highly sensitive method for genomewide detection of allelic composition in nonpaired, primary tumor specimens by use of affymetrix single-nucleotide-polymorphism genotyping microarrays. Am J Hum Genet 81: 114-126, 2007.
15. Lin DC, Meng X, Hazawa M, Nagata Y, Varela AM, Xu L, Sato Y, Liu LZ, Ding LW, Sharma A, et al: The genomic landscape of nasopharyngeal carcinoma. Nat Genet 46: 866-871, 2014.

16. Lin DC, Xu L, Ding LW, Sharma A, Liu LZ, Yang H, Tan P, Vadgama J, Karlan BY, Lester J, et al: Genomic and functional characterizations of phosphodiesterase subtype 4D in human cancers. Proc Natl Acad Sci USA 110: 6109-6114, 2013.

17. Liu Q, Huang J, Zhou N, Zhang Z, Zhang A, Lu Z, Wu F and Mo YY: lncRNA loc285194 is a p53-regulated tumor suppressor. Nucleic Acids Res 41: 4976-4987, 2013.

18. Lin DC, Xu L, Chen Y, Yan H, Hazawa M, Doan N, Said JW, Ding LW, Liu LZ, Yang H, et al: Genomic and functional analysis of the E3 ligase PARK2 in glioma. Cancer Res 75: 1815-1827, 2015.

19. Jiang YY, Lin DC, Mayakonda A, Hazawa M, Ding LW, Chien WW, Xu L, Chen Y, Xiao JF, Senapedis W, et al: Targeting super-enhancer-associated oncogenes in oesophageal squamous cell carcinoma. Gut: May 10, 2016 (Epub ahead of print). doi: 10.1136/gutjnl-2016-311818. 2016.

20. Pasic I, Shlien A, Durbin AD, Stavropoulos DJ, Baskin B, Ray PN, Novokmet A and Malkin D: Recurrent focal copynumber changes and loss of heterozygosity implicate two noncoding RNAs and one tumor suppressor gene at chromosome 3q13.31 in osteosarcoma. Cancer Res 70: 160-171, 2010.

21. Lin WM, Baker AC, Beroukhim R, Winckler W, Feng W, Marmion JM, Laine E, Greulich H, Tseng H, Gates C, et al: Modeling genomic diversity and tumor dependency in malignant melanoma. Cancer Res 68: 664-673, 2008.

22. Calhoun ES, Hucl T, Gallmeier E, West KM, Arking DE, Maitra A, Iacobuzio-Donahue CA, Chakravarti A, Hruban RH and Kern SE: Identifying allelic loss and homozygous deletions in pancreatic cancer without matched normals using highdensity single-nucleotide polymorphism arrays. Cancer Res 66: 7920-7928, 2006.

23. Nancarrow DJ, Handoko HY, Smithers BM, Gotley DC, Drew PA, Watson DI, Clouston AD, Hayward NK and Whiteman DC: Genome-wide copy number analysis in esophageal adenocarcinoma using high-density single-nucleotide polymorphism arrays. Cancer Res 68: 4163-4172, 2008

24. Teh MT, Blaydon D, Chaplin T, Foot NJ, Skoulakis S, Raghavan M, Harwood CA, Proby CM, Philpott MP, Young BD, et al: Genomewide single nucleotide polymorphism microarray mapping in basal cell carcinomas unveils uniparental disomy as a key somatic event. Cancer Res 65: 8597-8603, 2005.

25. Xue YL, Meng XQ, Ma LJ and Yuan Z: Plumbagin exhibits an anti-proliferative effect in human osteosarcoma cells by downregulating FHL2 and interfering with Wnt/ $\beta$-catenin signalling. Oncol Lett 12: 1095-1100, 2016.

26. Lv YF, Dai H, Yan GN, Meng G, Zhang $X$ and Guo QN: Downregulation of tumor suppressing STF cDNA 3 promotes epithelial-mesenchymal transition and tumor metastasis of osteosarcoma by the Wnt/GSK-3// $\beta$-catenin/Snail signaling pathway. Cancer Lett 373: 164-173, 2016.

27. Ng TL, Gown AM, Barry TS, Cheang MC, Chan AK, Turbin DA, Hsu FD, West RB and Nielsen TO: Nuclear beta-catenin in mesenchymal tumors. Mod Pathol 18: 68-74, 2005.

28. Yen CC, Chen WM, Chen TH, Chen WY, Chen PC, Chiou HJ, Hung GY, Wu HT, Wei CJ, Shiau CY, et al: Identification of chromosomal aberrations associated with disease progression and a novel 3q13.31 deletion involving LSAMP gene in osteosarcoma. Int J Oncol 35: 775-788, 2009.

29. Kresse SH, Ohnstad HO, Paulsen EB, Bjerkehagen B, Szuhai K, Serra M, Schaefer KL, Myklebost O and Meza-Zepeda LA: LSAMP, a novel candidate tumor suppressor gene in human osteosarcomas, identified by array comparative genomic hybridization. Genes Chromosomes Cancer 48: 679-693, 2009. 